\title{
Numerical Treatment of Singularly Perturbed Delay Differential Equations
}

\author{
K. Phaneendra ${ }^{1}$, Y.N. Reddy ${ }^{2}$, D. Kumara Swamy ${ }^{3}$ \\ ${ }^{I}$ (Department of Mathematics, Kakatiya Institute of Technology \& Science, Warangal-506014, India.) \\ ${ }^{2,3}$ (Department of Mathematics, National Institute of Technology, Warangal, 506004, India)
}

\begin{abstract}
In this paper, we propose numerical method to solve singularly perturbed delay differential equations which works smoothly in both the cases, i.e., whether the delay is of $O(\varepsilon)$ or of $o(\varepsilon)$. The numerical method uses the modified upwind finite difference scheme on a special type of mesh to tackle the delay argument. The stability and error analysis is given for in both the cases, when the sign of the coefficient of the reaction term is negative or positive. To demonstrate the efficiency of the method and how to discuss the size of the delay argument affects the layer behaviour we have implemented it on several test examples.
\end{abstract}

Keywords: Boundary Layer modified upwind finite difference scheme, Singular perturbation delay differential equation.

\section{INTRODUCTION}

A singularly perturbed delay differential equation is an ordinary differential equation in which the highest derivative is multiplied by a small parameter and involving at least one delay term. In the past, less attention had been paid for the numerical solution of singularly perturbed delay differential equations. But in recent years, there has been a growing interest in the numerical treatment of such differential equations. The literature on delay differential equations is mainly centered on first order initial value problems [1,2]. The boundary value problems of delay differential equations are ubiquitous in the variational problems in control theory [3]. For the numerical treatment for first order singularly perturbed delay differential equations, one can see the thesis by Tian [9]. Lange and Miura [6, 7] gave an asymptotic approach for a class of boundary-value problems for linear second-order differential-difference equations in which the highest order derivative is multiplied by small parameter and shows the effect of very small shifts (of the order of $\varepsilon$ ) on the solution and pointed out that they drastically affect the solution and therefore cannot be neglected. Kadalbajoo and Sharma [4], presented a numerical approach to solve singularly perturbed differential-difference equation, which contains only negative shift in the differentiated term. In this method they first approximate the shifted term by Taylor series and apply a difference scheme, provided shifts are $o(\varepsilon)$. Kadalbajoo and Sharma [5], presented a numerical method to solve singularly perturbed differential-difference equation which contains only negative shift not in the differentiated terms. In this method they present a numerical method composed of a standard upwind finite difference scheme on a special type of mesh shifts are either $o(\varepsilon)$ or $O(\varepsilon)$.

In this paper, we propose numerical method to solve singularly perturbed delay differential equations which works smoothly in both the cases, i.e., whether the delay is of $O(\varepsilon)$ or of $o(\varepsilon)$. The numerical method uses the modified upwind finite difference scheme on a special type of mesh to tackle the delay argument. The stability and error analysis is given for in both the cases, when the sign of the coefficient of the reaction term is negative or positive. To demonstrate the efficiency of the method and how to discuss the size of the delay argument affects the layer behaviour we have implemented it on several test examples.

\section{DESCRIPTION OF THE METHOD}

Consider a singularly perturbed delay differential equation

$\varepsilon y^{\prime \prime}(x)+a(x) y^{\prime}(x-\delta)+b(x) y(x)=f(x)$

on $0<\mathrm{x}<1,0<\delta<<1$,

with $y(x)=\phi(x), \quad-\delta \leq x \leq 0, \quad y(1)=\beta$,

where $a(x), b(x), f(x)$ are smooth functions, $\gamma$ is a constant and $\delta$ is the delay. For the function $y(x)$ be a smooth solution to the problem (1), it must satisfy boundary value problem be continuous on $[0,1]$ and be continuously differentiable on $(0,1)$. 
2.1. Layer on the left side Here, we consider the case $a(x) \geq M>0, \forall x \in[0,1]$, M being positive constant. In this case the solution of the boundary value problem exhibits boundary layer behaviour on the left side of the interval $[0,1]$ i.e., at $\mathrm{x}=0$

\section{Numerical Scheme}

In this section we construct a numerical scheme for solving the boundary value problem based on modified upwind finite difference scheme for the case when the solution of the problem exhibits a layer on the left side. The numerical method comprised a modified upwind finite difference operator on a special type of uniform mesh. To tackle the delay term, we choose the mesh parameter as $h=\frac{\delta}{m}$, where $m=p q, p$ is a positive integer and $q$ is the mantissa of $\delta$.

The modified upwind difference scheme of the boundary value problem (1) is given by $L^{N} y_{i}=\varepsilon D_{+} D_{-} y_{i}+a\left(x_{i}\right) \tilde{D}_{+} y_{i-m}+b\left(x_{i}\right) y_{i}=f_{i}$, for $\mathrm{i}=1,2,3 \ldots \mathrm{N}-1$,

$y_{i}=\phi_{i} \quad i=-m,-m+1 \ldots 0$

$y_{N}=\gamma$,

where

$$
D_{+} D_{-} y_{i}=\frac{\left(y_{i-1}-2 y_{i}+y_{i+1}\right)}{h^{2}}, D_{+} y_{i}=\frac{y_{i+1}-y_{i}}{h}, \tilde{D}_{+} y_{i}=\frac{y_{i+1}-y_{i}}{h}-\frac{h}{2} y_{i}^{\prime \prime}
$$

Then equation (3) gives

$$
\begin{aligned}
& \frac{\varepsilon}{h^{2}}\left(y_{i-1}-2 y_{i}+y_{i+1}\right)+b_{i} y_{i}= \\
& f_{i}-\frac{a_{i}}{h}\left(\phi_{i-m+1}-\phi_{i-m}\right)+\frac{a_{i}}{2 h}\left(\phi_{i-m+1}-2 \phi_{i-m}+\phi_{i-m-1}\right) \\
& \frac{\varepsilon}{h^{2}}\left(y_{m-1}-2 y_{m}+y_{m+1}\right)+\frac{a_{m}}{h} y_{i}+b_{m} y_{m}=f_{m}+\frac{a_{m}}{h} \phi_{0}+\frac{a_{m}}{2 h} \phi_{0}^{\prime \prime} \text { for } i=m \\
& \frac{\varepsilon}{h^{2}}\left(y_{i-1}-2 y_{i}+y_{i+1}\right)+a_{i}\left(\frac{\left(y_{i-m+1}-y_{i-m}\right)}{h}-\frac{h}{2} y_{i-m}^{\prime \prime}\right)+b_{i} y=f_{i} \text { for } i=m+1, m+2, \ldots . . N-1
\end{aligned}
$$

with $y_{0}=\phi_{0}$

$y_{N}=\gamma$

On simplification of the discrete problem (6), (7) and (8) reduces to a system of (N+1) linear difference equations given by $A^{N} y=f$

where $y=\left\langle y_{0,} y_{1}, \ldots \ldots y_{N}\right\rangle^{t}, f=\left\langle f_{0,} f_{1} \ldots \ldots . f_{N}\right\rangle^{t}$ and $A^{n}=\left[a_{i, j}\right], f\left(x_{i}\right)=f_{i} \quad$ for all $i=1,2, \ldots \ldots \ldots . . . N$ and $\phi\left(x_{i}\right)=\phi_{i}, \forall i=1,2,3, \ldots \ldots \ldots . . . \mathrm{m}$.

\section{II.1 Error estimate}

Case(i): When $\mathrm{b}(\mathrm{x}) \leq-\theta<0$, where $\theta$ is a positive constant

Lemma 2.1 (Discrete Minimum Principle). Suppose $\psi_{0} \geq 0$ and $\psi_{N} \geq 0$. Then $L^{N} \psi_{i} \leq 0$ for all $i$ $=1,2, \ldots \ldots . N-1$ implies $\psi_{i} \geq 0$ for all $i=0,1,2, \ldots \ldots, N$.

Proof. One can see [6]

Theorem 2.1: Under the assumptions that $\mathrm{a}(\mathrm{x}) \geq \mathrm{M}>0$ and $b(x) \leq-\theta<0$ where $\mathrm{M}$ and $\theta$ are positive constants, the solution of the discrete problem with boundary conditions exist, is unique and satisfies $\|y\|_{h, \infty} \leq \theta^{-1}\|f\|_{h, \infty}+C_{1}\left(\|\phi\|_{h, \infty}+|\gamma|\right)$

where $C_{1} \geq 1$ is a positive constant. Here, \|\|$_{h, \infty}$ is the discrete $l_{\infty}$ - norm defined by $\|x\|_{h, \infty}=\max _{0 \leq i \leq N}\left|x_{i}\right|$.

Proof. To prove the uniqueness and existence, suppose $\left\langle u_{i}\right\rangle_{i=0}^{N}$ and $\left\langle v_{i}\right\rangle_{i=0}^{N}$ be two solutions to the discrete problem then $z_{i}=u_{i}-v_{i}$ is a mesh function satisfying $z_{0}=0$ and for $1 \leq i \leq N-1$, we have $L^{N} z_{i}=L^{N} u_{i}-L^{N} v_{i}$. Since $u_{i}$ and $v_{i}$ satisfy therefore $L^{N} z_{i}=0,1 \leq i \leq N-1$, Thus the mesh function $z_{i}$ satisfies the hypothesis of the discrete minimum principle and so by an application of it to the mesh function $z_{i}$, 
we get $z_{i}=u_{i}-v_{i} \geq 0, \quad 0 \leq i \leq N$,

Again if we set $z_{i}=-\left(u_{i}-v_{i}\right)$, then $z_{i}$ is a mesh function satisfying $z_{0}=0=z_{N}$ and along the same lines we did above, we get $L^{N} z_{i}=0,1 \leq i \leq N-1$. Thus the application of the discrete minimum principle for the mesh function $z_{i}$ gives

$z_{i}=-\left(u_{i}-v_{i}\right) \geq 0$, i.e., $u_{i}-v_{i} \leq 0,0 \leq i \leq N$,

From equations (11) and (12), we get $u_{i}-v_{i}=0$, which implies the uniqueness of the solution to the discrete problem. For linear equations, the existence is implied by uniqueness. Now we shall prove the bound on $\left\langle y_{i}\right\rangle_{i=0}^{N}$. For that, we introduce two barrier functions $\psi_{i}^{ \pm}$defined by $\left.\psi_{i}^{ \pm}=\theta^{-1}\|f\|_{h, \infty}+C_{1}\|\phi\|+|\gamma|\right)_{ \pm} y_{i}$, $1 \leq i \leq N$, where $C_{1} \geq 1$ is an arbitrary positive constant.

Then we have $\psi_{0}^{ \pm}=\theta^{-1}\|f\|_{h, \infty}+C_{1}\left(\|\phi\|_{h, \infty}+|\gamma|\right) \pm y_{0}$

$=\theta^{-1}\|f\|_{h, \infty}+\left(C_{1}\|\phi\|_{h, \infty} \pm \phi_{0}\right)+C_{1}|\gamma|$, since $y_{0}=\phi_{0}$

$\geq 0$, since $\|\phi\|_{h, \infty} \geq \phi_{0}$ and $C_{1} \geq 1, \psi_{N}^{ \pm}=\theta^{-1}\|f\|_{h, \infty}+C_{1}\left(\|\phi\|_{h, \infty}+|\gamma|\right)_{ \pm} y_{N}$

$\theta^{-1}\|f\|_{h, \infty}+C_{1}\|\phi\|_{h, \infty}+\left(C_{1}|\gamma| \pm \gamma\right)$, since $y_{N}=\gamma \geq 0$, since $\|\gamma\| \geq \gamma$ and $C_{1} \geq 1$,

and for $0<i \leq m$, we have

$$
\begin{aligned}
L^{N} \psi_{i}^{ \pm} & =\varepsilon D_{+} D_{-} \psi_{i}^{ \pm}+a(x) D_{+} \psi_{i-m}^{ \pm}+b\left(x_{i}\right) \psi_{i}^{ \pm} \\
& =b\left(x_{i}\right)\left(\theta^{-1}\|f\|_{h, \infty}+\|\phi\|_{h, \infty}+|\gamma|\right)_{ \pm} L^{N} y_{i} .
\end{aligned}
$$

Using equation (5) for $L^{N} y_{i}$ in equation (13) we get Since $b\left(x_{i}\right) \leq-\theta<0$, i.e., $b\left(x_{i}\right) \theta^{-1} \leq-1$

$$
\begin{aligned}
& L^{N} \psi_{i}^{ \pm}=b\left(x_{i}\right)\left(\theta^{-1}\|f\|_{h, \infty}+\|\phi\|_{h, \infty}+|\gamma|\right) \\
& \pm\left(f_{i} .-a\left(x_{i}\right)\left(\phi_{i-m+1}-\phi_{i-m}\right) / h .+\frac{a_{i}}{2 h}\left(\phi_{i-m+1}-2 \phi_{i-m}+\phi_{i-m-1}\right)\right)
\end{aligned}
$$

we get

$$
\begin{aligned}
& L^{N} \psi_{i}^{ \pm} \leq\left(-\|f\|_{h, \infty} \pm f_{i}\right)+C_{1} b\left(x_{i}\right)\left(\|\phi\|_{h, \infty}+|\gamma|\right) \\
& \left.\quad\left(a\left(x_{i}\right)\left(\phi_{i-m+1}-\phi_{i-m}\right) / h\right)+\frac{a_{i}}{2 h}\left(\phi_{i-m+1}-2 \phi_{i-m}+\phi_{i-m-1}\right)\right)
\end{aligned}
$$

Since in the above inequality (15) the first and second terns are negative, so we choose the constant $C_{1}$ so that the sum of the moduli of the first and second terms dominates the modulus of the third term in the above inequality. We then obtain

$L^{N} \psi_{i}^{ \pm} \leq 0,1 \leq i \leq m$, for $m<i<N$,

We have

$$
\begin{aligned}
L^{N} \psi_{i}^{ \pm} & =\varepsilon D_{+} D_{-} \psi_{i}^{ \pm}+a(x) \psi_{i}^{ \pm}+b\left(x_{i}\right) \psi_{i}^{ \pm} \\
& =b\left(x_{i}\right)\left(\theta^{-1}\|f\|_{h, \infty}+C_{1}\left(\|\phi\|_{h, \infty}+|\gamma|\right)\right) \pm L^{N} y_{i} .
\end{aligned}
$$

Using equation (5) for $L^{N} y_{i}$, in equation (16) we get $L^{N} \psi_{i}^{ \pm}=b\left(x_{i}\right)\left(\theta^{-1}\|f\|_{h, \infty}+C_{1}\left(\|\phi\|_{h, \infty}+|\gamma|\right)\right) \pm f_{i}$

$$
\left.\leq\left(-\|f\|_{h, \infty} \pm f_{i}\right)+b\left(x_{i}\right) C_{1}\|\phi\|_{h, \infty}+|\gamma|\right) \text {, since } b\left(x_{i}\right) \theta^{-1} \leq-1<0 \text {, since } b\left(x_{i}\right) \leq-\theta<0,
$$

Combining both cases, we obtain $L^{N} \psi_{i}^{ \pm}<0,1<i<N$.

Using Lemma 2.1 for $\psi_{i}^{ \pm}$, obtain $\psi_{i}^{ \pm}=\theta^{-1}\|f\|_{h, \infty}+C_{1}(\|\phi\|+|\gamma|) \pm y_{i} \geq 0 \quad 1 \leq i \leq N$,

This proves the required estimate (10).

2.2. Case (ii): When $\mathrm{b}(\mathrm{x}) \geq \theta>0$

Lemma 2.2. (Discrete Maximum Principle): Suppose $\psi_{0} \geq 0$ and $\psi_{N} \geq 0$. Then $L^{N} \psi_{i} \geq 0$ for all $i=$ $1,2, \ldots . N-1$ implies that $\psi_{i} \geq 0$ for all $i=0,1,2, \ldots, N$.

Proof: One can see [6] 
Theorem 2.2. Under the assumption that $a(x) \geq M>0$ and $b(x) \geq \theta>0$, where $\mathrm{M}$ and $\theta$ are positive constants, the solutions of the discrete problem with boundary conditions exists, is unique and satisfies . $\|y\|_{h, \infty} \leq \theta^{-1}\|f\|_{h, \infty}+C_{2}\left(\|\phi\|_{h, \infty}+|\gamma|\right)$,

where $C_{2} \geq 1$ is a positive constant.

Proof. The proof of the uniqueness and existence is similar as in Theorem 2.1.Now we shall prove the bound on $\left\langle y_{i}\right\rangle_{i=0}^{N}$. For that, we introduce two barrier functions $\psi_{i}^{ \pm}$defined by $\psi_{i}^{ \pm}=\theta^{-1}\|f\|_{h, \infty}+C_{2}(\|\phi\|+|\gamma|) \pm y_{i}$, $0 \leq i \leq N$, where $C_{1} \geq 1$ is an arbitrary positive constant. Using the same argument as we used in the proof of Theorem 2.1, we obtain $\psi_{0} \geq 0, \psi_{N} \geq 0$ and for $0 \leq i \leq m$, we have $L^{N} \psi_{i}^{ \pm}=\varepsilon D_{+} D_{-} \psi_{i}^{ \pm}+a\left(x_{i}\right) D_{+} \psi_{i-m}^{ \pm}+b\left(x_{i}\right) \psi_{i}^{ \pm}=$ $b\left(x_{i}\right)\left(\theta^{-1}\|f\|_{h, \infty}+\|\phi\|_{h, \infty}+|\gamma|\right) \pm L^{N} y_{i}$.

$$
L^{N} \psi_{i}^{ \pm}=b\left(x_{i}\right)\left(\theta^{-1}\|f\|_{h, \infty}+\|\phi\|_{h, \infty}+|\gamma|\right) \pm
$$

Using equation (5) we get $\left(\begin{array}{l}f_{i}-a\left(x_{i}\right)\left(\phi_{i-m+1}-\phi_{i-m}\right) / h \\ \left.+a\left(x_{i}\right)\left(\phi_{i-m+1}-\phi_{i-m}\right) / h\right)+\frac{a_{i}}{2 h}\left(\phi_{i-m+1}-2 \phi_{i-m}+\phi_{i-m-1}\right)\end{array}\right)$ Since $b\left(x_{i}\right) \geq \theta>0$, i.e., $b\left(x_{i}\right) \theta^{-1} \geq 1$, we get

$$
\begin{aligned}
L^{N} \psi_{i}^{ \pm} & \leq\left(\|f\|_{h, \infty}+f_{i}\right) \pm C_{2} b\left(x_{i}\right)\left(\|\phi\|_{h, \infty}+|\gamma|\right) \\
& \left.\mp\left(a\left(x_{i}\right)\left(\phi_{i-m+1}-\phi_{i-m}\right) / h\right)+\frac{a_{i}}{2 h}\left(\phi_{i-m+1}-2 \phi_{i-m}+\phi_{i-m-1}\right)\right)
\end{aligned}
$$

Since $\|f\|_{h, \infty} \geq f_{i}$ in the above inequality the first and second terns are positive, so we choose the constant $C_{2}$ such that the sum of the moduli of the first and second terms dominates the modulus of the third term in the above inequality. We then obtain

$$
L^{N} \psi_{i}^{ \pm} \geq 0
$$

For $m<i<N$, we have $L^{N} \psi_{i}^{ \pm}=\varepsilon D_{+} D_{-} \psi_{i}^{ \pm}+a(x) \psi_{i}^{ \pm}+b\left(x_{i}\right) \psi_{i}^{ \pm}$

$$
=b\left(x_{i}\right)\left(\theta^{-1}\|f\|_{h, \infty}+C_{2}\left(\|\phi\|_{h, \infty}+|\gamma|\right)\right) \pm L^{N} y_{i} \text {. }
$$

Using Eq. (5), we get

$$
\begin{aligned}
L^{N} \psi_{i}^{ \pm}= & b\left(x_{i}\right)\left(\theta^{-1}\|f\|_{h, \infty}+C_{2}\left(\|\phi\|_{h, \infty}+|\gamma|\right)\right) \pm f_{i} \\
& =b\left(x_{i}\right)\left(\theta^{-1}\|f\|_{h, \infty} \pm f_{i}\right)+b\left(x_{i}\right)\left(\|\phi\|_{h, \infty}+|\gamma|\right)>0 \text { since } b\left(x_{i}\right) \geq \theta>0
\end{aligned}
$$

From inequalities (20) and (21) we get

$$
L^{N} \psi_{i}^{ \pm} \geq 0,1<i<N .
$$

Thus an application of Lemma 2.2 to mesh function $\psi_{i}^{ \pm}$gives $\psi_{i}^{ \pm}=\theta^{-1}\|f\|_{h, \infty}+C_{2}(\|\phi\|+|\gamma|) \pm y_{i} \geq 0 \quad 0 \leq i \leq N$, which proves the required bound on the discrete solution $\left\langle y_{i}\right\rangle_{i=0}^{N}$.

Thus theorems (2.1) and (2.2) imply that the solution to the discrete problem (1.1), (1.2) is uniformly bounded, independently of the mesh parameter $h$ and the parameter $\varepsilon$, which proves the difference scheme is stable for all mesh sizes.

Corollary2.1. Under the assumption that $a\left(x_{i}\right) \geq M>0$, the error $e_{i}=y\left(x_{i}\right)-y_{i}$ between the solution $y\left(x_{i}\right)$ of the continuous problem (1) and the solution $y_{i}$ of the discrete problem (1) and (2) satisfies the estimate $\|e\|_{h, \infty} \leq \theta^{-1}\|T\|_{h, \infty}$

where $T_{i}$ satisfies

$$
T_{i} \leq\left[\begin{array}{l}
\frac{h^{2}}{12} \max _{x_{i-1} \leq x \leq x_{i+1}}\left|y^{i v(x)}\right|+ \\
\|a\| \|_{x_{i-m} \leq x \leq x_{i-m+1}}\left\{\frac{h^{2}}{6}\left|y^{\prime \prime \prime}(x)\right|+\frac{h^{3}}{24}\left|y^{i v}(x)\right|\right\}
\end{array}\right] .
$$


Proof: The truncation error $T_{i}$ is given by

$$
\begin{aligned}
T_{i} & =\varepsilon\left[\left(y_{i-1}-2 y_{i}+y_{i+1}\right) / h^{2}-y^{\prime \prime}\left(x_{i}\right)\right] \\
& +a\left(x_{i}\right)\left[\frac{\left(y_{i-m+1}-y_{i-m}\right)}{h}-\frac{h}{2} y^{\prime \prime}\left(x_{i-m}\right)-y_{i-m}^{\prime}\right] .
\end{aligned}
$$

Now using the Taylor's series and after some simplifications, we obtain

$$
T_{i} \leq\left[\begin{array}{ll}
\frac{\varepsilon h^{2}}{12} & \max _{x_{i-1} \leq x \leq x_{i+1}}\left|y^{i v(x)}\right|+ \\
\|a\| & \max _{x_{i-m} \leq x \leq x_{i-m+1}}\left\{\frac{h^{2}}{6}\left|y^{\prime \prime \prime}(x)\right|+\frac{h^{3}}{24}\left|y^{i v}(x)\right|\right\}
\end{array}\right] .
$$

We have

$L^{N} e\left(x_{i}\right)=L^{N} y\left(x_{i}\right)-L^{N} y_{i}=T_{i}$, where $\mathrm{i}=1,2,3, \ldots \mathrm{N}-1 \quad$ and $e_{0}=0=e_{N}$.

Then by using theorems (2.1) and (2.2), we obtain the required error estimate

\section{LAYER ON THE RIGHT SIDE}

we consider the case $a(x) \leq-M<0$, M being positive constant i.e., the solution of the boundary value problem exhibits boundary layer behaviour on the right side of the interval $[0,1]$ i.e., at $x=1$ and construct a numerical scheme to solve the problem.

\section{Numerical Scheme}

The modified upwind difference scheme of the boundary value problem (1) and (2) is given byl

$$
\begin{aligned}
& L^{N} y_{i}=\varepsilon D_{+} D_{-} y_{i}+a\left(x_{i}\right) \tilde{D}_{-} y_{i-m}+b\left(x_{i}\right) y_{i}=f_{i}, \\
& y_{i}=\phi_{i} \quad i=-m,-m+1, . ., 0 \\
& y_{N}=\gamma
\end{aligned}
$$

where $D_{+} D_{-} y_{i}=\frac{\left(y_{i-1}-2 y_{i}+y_{i+1}\right)}{h^{2}}, D_{-} y_{i}=\frac{y_{i}-y_{i-1}}{h} \tilde{D}_{-} y_{i}=\frac{y_{i}-y_{i-1}}{h}+\frac{h}{2} y_{i}^{\prime \prime}$

The equation (24) gives the system of equations

$$
\begin{aligned}
& \frac{\varepsilon}{h^{2}}\left(y_{i-1}-2 y_{i}+y_{i+1}\right)+b_{i} y_{i}=f_{i}-\frac{a_{i}}{h}\left(\phi_{i-m}-\phi_{i-m-1}\right) \\
& \text { for } i=1,2,3 \ldots m-1 \\
& -\frac{a_{i}}{2 h}\left(\phi_{i-m+1}-2 \phi_{i-m}+\phi_{i-m-1}\right) \\
& \frac{\varepsilon}{h^{2}}\left(y_{m-1}-2 y m+y_{m+1}\right)+\frac{a_{m}}{h} y_{0}+b_{m} y_{m}=f_{m}+\frac{a_{m}}{h} \phi_{0}-\frac{a_{m} h}{2} \phi_{0}^{\prime \prime} \\
& \text { for } i=m \\
& \frac{\varepsilon}{h^{2}}\left(y_{i-1}-2 y_{i}+y_{i+1}\right)+a_{i}\left(\frac{\left(y_{i-m}-y_{i-m-1}\right)}{h}+\frac{h}{2} y_{i-m}^{\prime}\right)+b_{i} y=f_{i} \text { for } i=m+1, m+2, \ldots . N-1 \\
& y_{0}=\phi_{0} \text {, } \\
& y_{N}=\gamma
\end{aligned}
$$

On simplification the discrete problem reduces to a system of $(N+1)$ linear difference equations given by $A^{N} y=f$, where $\mathrm{y}=\left\langle y_{0}, y_{1,} \ldots \ldots y_{N}\right\rangle^{t}, \mathrm{f}=\left\langle f_{0,} f_{1,} \ldots \ldots f_{N}\right\rangle^{t}$ and $A^{n}=\left[a_{i, j}\right]$,

IV.

NUMERICAL EXAMPLES

To describe the method we consider six test examples with left and right end boundary layers.

Example 1. $\varepsilon y^{\prime \prime}(x)+y^{\prime}(x-\delta)-y(x)=0$ under the interval with boundary conditions $y(x)=1,-\delta \leq x \leq 0$, $y(1)=0$

Example 2. $\varepsilon y^{\prime \prime}(x)+0.25 y^{\prime}(x-\delta)-y(x)=0$ under the interval with boundary conditions $\quad y(x)=1$, $-\delta \leq x \leq 0, \quad y(1)=0$

Example3.[6, p. 257] $\varepsilon y^{\prime \prime}(x)-y^{\prime}(x-\delta)-y(x)=0$ under the interval with boundary conditions $y(x)=1$, $-\delta \leq x \leq 0, \quad y(1)=-1$

Example 4. $\varepsilon y^{\prime \prime}(x)+0.25 y^{\prime}(x-\delta)-y(x)=0$ under the interval with boundary conditions $\mathrm{y}(\mathrm{x})=1,-\delta \leq x \leq 0$, $y(1)=-1$ 
Example 5. [7, 275] $\varepsilon y^{\prime \prime}(x)+y^{\prime}(x-\delta)+y(x)=0$ under the interval with boundary conditions $\mathrm{y}(\mathrm{x})=1$, $-\delta \leq x \leq 0, \quad \mathrm{y}(1)=1$

Example 6. $\varepsilon y^{\prime \prime}(x)-y^{\prime}(x-\delta)+y(x)=0$ under the interval with boundary conditions $\mathrm{y}(\mathrm{x})=1,-\delta \leq x \leq 0$, $\mathrm{y}(1)=-1$

Table 1. The maximum absolute errors for $\varepsilon=0.1$

$\begin{array}{llllll}\delta \backslash N & 100 & 200 & 300 & 400 & 500\end{array}$

\section{Example 1}

$\begin{array}{llllll}0.03 & 0.010738 & 0.005458 & 0.0041115 & 0.002542 & 0.0020691\end{array}$

$\begin{array}{llllll}0.05 & 0.010711 & 0.005452 & 0.0041079 & 0.002541 & 0.0020679\end{array}$

$\begin{array}{llllll}0.08 & 0.010672 & 0.005441 & 0.0041008 & 0.002537 & 0.0020655\end{array}$

Example 5

$\begin{array}{llllll}0.03 & 0.009977 & 0.004899 & 0.003386 & 0.002504 & 0.001987\end{array}$

$\begin{array}{llllll}0.05 & 0.009961 & 0.004903 & 0.003391 & 0.002509 & 0.001991\end{array}$

$\begin{array}{llllll}0.08 & 0.009969 & 0.004923 & 0.003406 & 0.002522 & 0.002002\end{array}$

Example 6

$0.03 \quad 5.809 \mathrm{e}-005 \quad 1.443 \mathrm{e}-005 \quad 6.689 \mathrm{e}-006 \quad 3.715 \mathrm{e}-005 \quad 2.359$ e-006

$0.05 \quad 9.872 \mathrm{e}-005 \quad 2.420 \mathrm{e}-005 \quad 1.118 \mathrm{e}-005 \quad 6.192 \mathrm{e}-006 \quad 3.922 \mathrm{e}-006$

$0.08 \quad 1.614 \mathrm{e}-004 \quad 3.877 \mathrm{e}-005 \quad 1.788 \mathrm{e}-005 \quad 9.843 \mathrm{e}-006 \quad 6.205 \mathrm{e}-006$

Table 2. The maximum absolute errors for $\delta=0.03$

\begin{tabular}{ccccc}
\hline$\varepsilon \backslash N$ & 100 & 200 & 300 & 400 \\
\hline \multicolumn{5}{c}{ Example 2 } \\
$2^{-1}$ & 0.010026 & 0.004905 & 0.003387 & 0.002504 \\
$2^{-2}$ & 0.010018 & 0.004903 & 0.003386 & 0.002503 \\
$2^{-3}$ & 0.010004 & 0.004898 & 0.003383 & 0.002501 \\
$2^{-4}$ & 0.009974 & 0.004888 & 0.003377 & 0.002497 \\
$2^{-5}$ & 0.009916 & 0.004867 & 0.003366 & 0.002489 \\
$2^{-6}$ & 0.009798 & 0.004827 & 0.003343 & 0.002473 \\
$2^{-7}$ & 0.009559 & 0.004745 & 0.003296 & 0.002441
\end{tabular}

Example 3

$2^{-1} \quad 1.1301 \mathrm{e}-005 \quad 2.8562 \mathrm{e}-006 \quad 1.3285 \mathrm{e}-006 \quad 7.4052 \mathrm{e}-007$

$2^{-2} \quad 2.2807 \mathrm{e}-005 \quad 5.7406 \mathrm{e}-006 \quad 2.6661 \mathrm{e}-006 \quad 1.4851 \mathrm{e}-006$

$2^{-3} \quad 4.6429 \mathrm{e}-005 \quad 1.1594 \mathrm{e}-005 \quad 5.3679 \mathrm{e}-006 \quad 2.9869 \mathrm{e}-006$

$\begin{array}{lllll}2^{-4} & 9.6118 \mathrm{e}-005 & 2.3641 \mathrm{e}-005 & 1.0878 \mathrm{e}-005 & 6.0402 \mathrm{e}-006\end{array}$

$2^{-5} \quad 2.0526 \mathrm{e}-004 \quad 4.9100 \mathrm{e}-005 \quad 2.2331 \mathrm{e}-005 \quad 1.2346 \mathrm{e}-005$

$2^{-6} \quad 4.6264 \mathrm{e}-004 \quad 1.0551 \mathrm{e}-004 \quad 4.6976 \mathrm{e}-005 \quad 2.5763 \mathrm{e}-005$

$\begin{array}{llllll}2^{-7} & 0.001133 & 2.4061 \mathrm{e}-004 & 1.0333 \mathrm{e}-004 & 5.5864 \mathrm{e}-005\end{array}$

Example 4

$2^{-1} \quad 2.8006 \mathrm{e}-007 \quad 7.1060 \mathrm{e}-008 \quad 3.3105 \mathrm{e}-008 \quad 1.8462 \mathrm{e}-008$

$2^{-2} \quad 5.6024 \mathrm{e}-007 \quad 1.4213 \mathrm{e}-007 \quad 6.6216 \mathrm{e}-008 \quad 3.6927 \mathrm{e}-008$

$2^{-3} \quad 1.1209 \mathrm{e}-006 \quad 2.8433 \mathrm{e}-007 \quad 1.3245 \mathrm{e}-007 \quad 7.3863 \mathrm{e}-008$

$2^{-4} \quad 2.2438 \mathrm{e}-006 \quad 5.6892 \mathrm{e}-007 \quad 2.6498 \mathrm{e}-007 \quad 1.4776 \mathrm{e}-007$

$2^{-5} \quad 4.4954 \mathrm{e}-006 \quad 1.1388 \mathrm{e}-006 \quad 5.3028 \mathrm{e}-007 \quad 2.9566 \mathrm{e}-007$

$2^{-6} \quad 9.0217 \mathrm{e}-006 \quad 2.2817 \mathrm{e}-006 \quad 1.0618 \mathrm{e}-006 \quad 5.9187 \mathrm{e}-007$

$2^{-7} \quad 1.8167 \mathrm{e}-005 \quad 4.5797 \mathrm{e}-006 \quad 2.1287 \mathrm{e}-006 \quad 1.1859 \mathrm{e}-006$ 


\section{DISCUSSIONS AND CONCLUSIONS}

The maximum absolute errors are presented in tables 1 and 2 for the considered examples in support of the proposed method to show the effect of delay on the boundary layer behaviour of the solution. We have presented maximum absolute errors by the double mesh principle given by $Z_{h}=\max _{j}\left|y_{j}^{h}-y_{j}^{h / 2}\right|, j=0,1, \ldots . .$, $N-1$ where $y_{j}^{h}$ is the computed solution on the mesh $\left\{x_{j}\right\}_{0}^{N}$ at the nodal point $x_{j}$ where and $y_{j}^{h / 2}$ is the computed solution at the nodal point $x_{j}$ on the mesh $\left\{x_{j}\right\}_{0}^{2 N}$ where $x_{j}=x_{j-1}+h / 2, j=1(1) 2 N$. The existence and uniqueness of the discrete problem along with stability estimates are discussed.

We observe that if $\delta=o(\varepsilon)$ and as $\delta$ increases, the thickness of the boundary layer decreases in the case when the solution exhibits layer behaviour on the left side, while in the case of the right side boundary layer, it increases. This method gives good.

\section{References}

[1] R. Bellman, K. L. Cooke, Differential-Difference Equations, Academic Press, New York, USA, 1963.

[2] R. D. Driver, Ordinary and Delay Differential Equations, Belin-Heidelberg, New York, Springer, 1977.

[3] V. Y. Glizer, Asymptotic solution of a boundary-value problem for linear singularly-perturbed functional differential equations arising in optical control theory, J. Optim. Theory Appl. 106 (2000) 309-335.

[4] M. K. Kadalbajoo, K. K. Sharma, Numerical analysis of singularly perturbed delay differential equations with layer behavior, Applied Mathematics and Computation, 157 (2004) 11-28.

[5] M. K. Kadalbajoo, K. K. Sharma, Numerical treatment of boundary value problems for second order singularly perturbed delay differential equations, Computational \& Applied Mathematics, 24 (2005) 151-172.

[6] M. K. Kadalbajoo, K. K. Sharma, A Numerical method based on finite differences for boundary value problems for singularly perturbed delay differential equations, Applied Mathematics \& Computation, 197 (2008) 692-707.

[7] C. G. Lange, R. M. Miura, Singular perturbation analysis of boundary-value problems for differential-difference equations. v. small shifts with layer behavior, SIAM J. Appl. Math. 54 (1994) 249-272.

[8] C. G. Lange, \& R. M. Miura, Singular perturbation analysis of boundary-value problems for differential-difference equations. vi. Small shifts with rapid oscillations, SIAM J. Appl. Math. 54 (1994) 273-283.

[9] M. K. Kadalbazoo, Y. N. Reddy, A non asymptotic method for general linear singular perturbation problems, J. Optim. Theory Appl. 55 (1986) 439-452.

[10] H. Tian, Numerical treatment of singularly perturbed delay differential equations, Ph.D. thesis, University of Manchester, 2000. 